\title{
ForestHash: Semantic Hashing With Shallow Random Forests and Tiny Convolutional Networks
}

\author{
Qiang Qiu ${ }^{1}$, José Lezama ${ }^{2}$, Alex Bronstein ${ }^{3}$, and Guillermo Sapiro ${ }^{1}$ \\ 1 Duke University, USA \\ 2 Universidad de la República, Uruguay \\ 3 Technion-Israel Institute of Technology, Israel
}

\begin{abstract}
In this paper, we introduce a random forest semantic hashing scheme that embeds tiny convolutional neural networks $(\mathrm{CNN})$ into shallow random forests. A binary hash code for a data point is obtained by a set of decision trees, setting ' 1 ' for the visited tree leaf, and ' 0 ' for the rest. We propose to first randomly group arriving classes at each tree split node into two groups, obtaining a significantly simplified two-class classification problem that can be a handled with a light-weight CNN weak learner. Code uniqueness is achieved via the random class grouping, whilst code consistency is achieved using a low-rank loss in the CNN weak learners that encourages intra-class compactness for the two random class groups. Finally, we introduce an information-theoretic approach for aggregating codes of individual trees into a single hash code, producing a nearoptimal unique hash for each class. The proposed approach significantly outperforms state-of-the-art hashing methods for image retrieval tasks on large-scale public datasets, and is comparable to image classification methods while utilizing a more compact, efficient and scalable representation. This work proposes a principled and robust procedure to train and deploy in parallel an ensemble of light-weight CNNs, instead of simply going deeper.
\end{abstract}

\section{Introduction}

In view of the recent huge interest in image classification and object recognition problems and the spectacular success of deep learning and random forests in solving these tasks, modest efforts are being invested into the related, and often more difficult, problems of image and multimodal content-based retrieval, and, more generally, similarity assessment in very large-scale databases. These problems, arising as primitives in many computer vision tasks, are becoming increasingly important in the era of exponentially increasing information. Semantic and similarity-preserving hashing methods have recently received considerable attention for addressing such a need, in part due to their significant memory and computational advantage over other representations. These methods learn to embed data points into a space of binary strings; thus producing compact representations with constant or sub-linear search time; this is critical and one of the few options for low-cost truly big data. Such an embedding can be considered as a hashing function on the data, which translates the underlying similarity into the collision probability of the hash or, more generally, into the similarity of the codes under the Hamming metric. Examples of recent similarity-preserving hashing methods include 


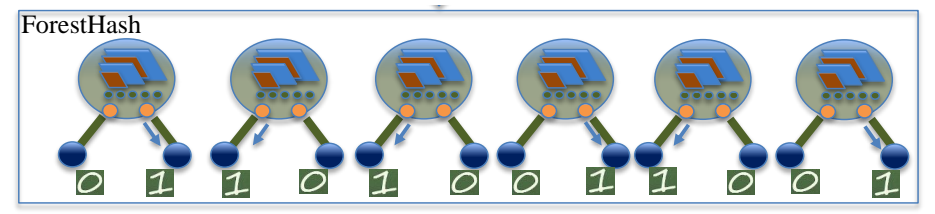

Fig. 1. ForestHash embeds tiny convolutional neural networks (CNN) into shallow random forests. ForestHash consists of shallow random trees in a forest, usually of depth 2 or 3 . At each tree split node, arriving classes are randomly partitioned into two groups for a significantly simplified two-class classification problem, which can be sufficiently handled by a light-weight CNN weak learner, usually of 2 to 4 layers. We set 1 for the visited tree leaf, and 0 for the rest. By simultaneously pushing each data point through $M$ trees of the depth $d$, we obtain $M\left(2^{d-1}\right)$-bit hash codes. The random grouping of the classes enables code uniqueness by enforcing that each class shares code with different classes in different trees. The non-conventional low-rank loss adopted for CNN weak learners encourages code consistency by minimizing intra-class variations and maximizing inter-class distance for the two random class groups. The obtained ForestHash codes serve as efficient and compact image representation for both image retrieval and classification.

Locality-Sensitive Hashing (LSH) [1] and its kernelized version (KLSH) [2], Spectral Hashing (SH) [3], Sparse Hash [4], Kernel-based Supervised Hashing (KSH) [5], Anchor Graph Hashing (AGH) [6], Self-Taught Hashing (STH) [7], and Deep Supervised Hashing (DSH) [8].

Due to the profound similarity between the problems of semantic hashing and that of binary classification, numerous classification techniques have been adapted to the former task. For example, multiple state-of-the-art supervised hashing techniques like ANN Hashing [9], SparseHash [4], HDML [10] and DSH [7] are based on deep learning methodologies. Besides deep learning, random forest [11,12] is another popular classification technique that has recently shown great success for a large variety of classification tasks, such as pose estimation [13] and object recognition [14]. However, to the best of our knowledge, random forests have not been used so far to construct semantic hashing schemes, and therefore do not enjoy the advantages of such compact and efficient codes. This is mainly because acting as hashing functions, a random forest fails to preserve the underlying similarity due to the inconsistency of hash codes generated in each tree for the same class data; it also lacks a principled way of aggregating hash codes produced by individual trees into a single longer code.

In this paper, we propose the ForestHash scheme. As shown in Figure 1 the proposed ForestHash is designed to provide consistent and unique hashes to images from the same semantic class, by embedding tiny convolutional neural networks (CNN) into shallow random forests. We start with a simple hashing scheme, where random trees in a forest act as hashing functions by setting ' 1 ' for the visited tree leaf, and ' 0 ' for the rest. To enable such hashing scheme, we first introduce random class grouping to randomly partition arriving classes into two groups at each tree split node. The class random grouping enables code uniqueness by enforcing each class to share code with different classes in different trees, and also produces a significantly reduced two-class problem being sufficiently handled by a light-weight CNN. 
We further adopt a non-conventional low-rank loss for CNN weak learners to encourage code consistency by minimizing intra-class variations and maximizing interclass distance for the two random class groups, thereby preserving similarity. The lowrank loss is based on the assumption that high-dimensional data often have a small intrinsic dimension. Consequently, when data from the same low-dimensional subspace are arranged as columns of a single matrix, this matrix should be approximately lowrank. In Section 2.3, we show how to learn a linear transformation of subspaces using the matrix nuclear norm as the optimization criterion. We discuss both experimentally and theoretically that such learned transformation simultaneously minimizes intra-class variation and maximizes inter-class separation. We further show that kernelization or deep learning can be used to handle intricate data that do not necessarily admit a linear model.

Finally, the proposed information-theoretic aggregation scheme provides a principled way to combine hashes from each independently trained random tree in the forest. The aggregation process discussed in Section 2.4 is performed efficiently in a greedy way, which still achieves a near-optimal solution due to submodularity of the mutual information criterion being optimized. We discuss both unsupervised and supervised hash aggregation.

In Section 3, we show a comprehensive experimental evaluation of the proposed representation scheme, demonstrating that it significantly outperforms state-of-the-art hashing methods for large-scale image and multi-modal retrieval tasks.

\section{Forest hashing}

We first discuss a simple random forest hashing scheme, where independently trained random trees act as hashing functions by setting ' 1 ' for the visited tree leaf, and ' 0 ' for the rest. We also show that hashes from a forest often fail to preserve the desired intra-class similarity.

\subsection{A toy hashing scheme}

Random forest [11,12] is an ensemble of binary decision trees, where each tree consists of hierarchically connected split (internal) nodes and leaf (terminal) nodes. Each split node corresponds to a weak learner, and evaluates each arriving data point sending it to the left or right child based on the weak learner binary outputs. Each leaf node stores the statistics of the data points that arrived to it during training. During testing, each decision tree returns a class posterior probability for a test sample, and the forest output is often defined as the average (or otherwise aggregated distribution) of the tree posteriors.

Following the random forest literature [12], in this paper, we specify a maximum tree depth $d$ to limit the size of a tree, which is different from algorithms like C4.5 [15] that grow the tree relying on other termination criteria; we also avoid post-training operations such as tree pruning. Thus, a tree of depth $d$ consists of $2^{d-1}$ tree leaf nodes, indexed in the breadth-first order. 
During training, we can introduce randomness into the forest through a combination of random set sampling and randomized node optimization, thereby avoiding duplicate trees. As discussed in [11,12], training each tree with a different randomly selected set decreases the risk of overfitting, improves the generalization of classification forests, and significantly reduces the training time. When given more than two classes, we introduce node randomness by randomly partitioning the classes arriving at each binary split node into two categories.

A toy pedagogic hashing scheme is constructed as follows: Each data point is pushed through a tree until reaching the corresponding leaf node. We simply set ' 1 ' for leaf nodes visited, and ' 0 ' for the rest. By ordering those bits in a predefined node order, e.g., the breadth-first order, we obtain a $\left(2^{d-1}\right)$-bit hash code, always containing exactly one 1 . In a random forest consisting of $M$ trees of the depth $d$, each point is simultaneously pushed through all trees to obtain $M\left(2^{d-1}\right)$-bit hash codes.

This hashing scheme has several obvious characteristics and advantages: First, both the training and the hashing processes can be done in parallel to achieve high computational efficiency on modern parallel CPU or GPU hardware. Second, multiple hash codes obtained from a forest, each from an independently trained tree, have the potential to inherit the boosting effect of the random forest, i.e., increasing the number of trees increases accuracy (sublinearly) [12]. Finally, the scheme guarantees 1-sparsity for hash codes from each tree.

However, hashes from a forest fail to preserve the underlying data similarity. In classification, for which the forest was originally designed, an ensemble posterior is obtained by averaging from a large number of trees, thus boosting the classification accuracy [11], and no confident class posteriors are required for individual trees. This has several negative consequences for constructing a suitable hash function. First, a forest often distributes same class samples over multiple leave nodes in a tree, thus, no consistent codes are assigned to each class. Second, for the same reason, samples of different classes can follow the same path, therefore a forest does not guarantee a unique code for each class. Moreover, it is not obvious how to combine hashes from different trees given a target code length.

The inconsistency of the hash codes becomes more severe when increasing the tree depth, as more leaf nodes are available to distribute the same class samples. This problem can not be solved by simply increasing the number of trees for longer total bit length. For example, if 4-bit inconsistency is allowed for a 64-bit hash code, the Hamming ball already contains $C_{64}^{4}=635,376$ codes. A principled way is required to combine hashes from each tree. One can choose to combine hashes from different trees simply through concatenating, averaging and thresholding, or voting. However, the principles behind those heuristics are not obvious, and we might loose control on code length, sparsity, and even binarity.

In what follows, we address these two problems. First, we propose the random class grouping scheme, followed with near-optimal code aggregation, to enforce code uniqueness for each class. Second, we adopt a non-conventional low-rank loss for weak learners to encourage code consistency. 


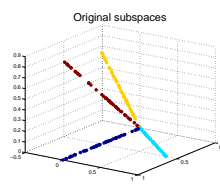

(a)

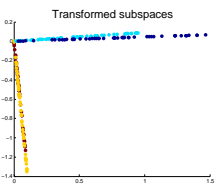

(b)

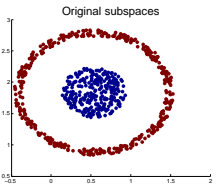

(c)

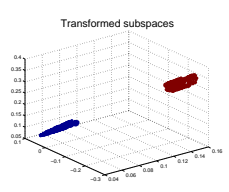

(d)

Fig. 2. Synthetic two-class examples illustrating the properties of the learned low-rank transformation. (a), (c) are transformed to (b), (d), respectively. In (a), two classes are defined as \{blue, cyan $\}$ and $\{$ yellow, red $\}$. An RBF kernel is applied to transform (c) to (d)

\subsection{Random class grouping}

A random class grouping scheme is first introduced to randomly partition arriving classes into two groups at each tree split node. Random class grouping serves two main purposes: First, a multi-class problem is significantly reduced to a two-class classification problem at each split node, which can be sufficiently handled by a very light-weight CNN weak learner. Second, random class grouping enforces each class to share its code with different classes in different trees, which allows the information-theoretic aggregation developed in the sequel to later produce a near-optimal unique hash code for each class.

\subsection{Low-rank loss}

A non-conventional low-rank loss is adopted for weak learners, e.g., a light-weight $\mathrm{CNN}$ learner, in a forest. Consider $s$-dimensional data points belonging to two classes after random class grouping, which for simplicity are denoted as positive and negative. We stack the points as columns of the matrices $\mathbf{X}^{+}$and $\mathbf{X}^{-}$, respectively. Let $\|\mathbf{A}\|_{*}$ denote the nuclear norm of the matrix $\mathbf{A}$, i.e., the sum of its singular values. The nuclear norm is known to be the convex envelope of matrix rank over the unit ball of matrices [16]. The following result in [17] helps motivate our per-node classifier:

Lemma 1. Let $\mathbf{A}$ and $\mathbf{B}$ be matrices of the same row dimensions, and $[\mathbf{A}, \mathbf{B}]$ denote their column-wise concatenation. Then, $\|[\mathbf{A}, \mathbf{B}]\|_{*} \leq\|\mathbf{A}\|_{*}+\|\mathbf{B}\|_{*}$, with equality holding if the column spaces of $\mathbf{A}$ and $\mathbf{B}$ are orthogonal.

At each tree split node, we propose to learn a weight matrix $\mathbf{W}$ minimizing the following low-rank loss function.

$$
\min _{\mathbf{W}}\left\|\mathbf{W} \mathbf{X}^{+}\right\|_{*}+\left\|\mathbf{W} \mathbf{X}^{-}\right\|_{*}-\left\|\mathbf{W}\left[\mathbf{X}^{+}, \mathbf{X}^{-}\right]\right\|_{*},
$$

Based on Lemma 1, the loss function (1) reaches its minimum 0 if the column spaces of the two classes become orthogonal after applying the learned transformation $\mathbf{W}$. Equivalently, (1) reaches the minimum 0 if the subspaces of the two classes are maximally opened up after transformation, i.e., the smallest principal angle between the subspaces 
equals $\frac{\pi}{2}$. Simultaneously, minimizing the first two nuclear norm terms in 11 helps reduce the variation within classes. Synthetic examples presented in Figure 2 illustrate the properties of the learned transformation. The trivial solution $\mathbf{W}=0$ can be avoided through a good initialization, e.g., the identity matrix [17].

Splitting functions. With random class grouping, we have a two-class classification problem at each split node. We stack the training data points from each class as columns of the matrices $\mathbf{X}^{+}$and $\mathbf{X}^{-}$, respectively.

During training, at the $i$-th split node, we denote the arriving training samples as $\mathbf{X}^{+}$and $\mathbf{X}^{-}$. After a weight matrix $\mathbf{W}$ is successfully learned by minimizing 11 , it is reasonable to assume that each of the classes will belong to a low-dimensional subspace, the distance from which can be used to classify previously unseen points. We use $k$ SVD [18] to learn a pair of dictionaries $\mathbf{D}^{ \pm}$, for each of the two classes, by minimizing

$$
\min _{\mathbf{D}_{ \pm}, \mathbf{Z}^{ \pm}}\left\|\mathbf{W} \mathbf{X}^{ \pm}-\mathbf{D}^{ \pm} \mathbf{Z}^{ \pm}\right\| \text {s.t. }\left\|\mathbf{z}^{ \pm}\right\|_{0} \leq l,
$$

where the $\ell_{0}$ pseudonorm $\left\|\mathbf{z}^{ \pm}\right\|_{0}$ counts the number of non-zero elements in each column of $\mathbf{Z}^{ \pm}$, and $l$ controls the subspace dimension.

At testing, given a data point $\mathbf{x}$, the splitting function is evaluated by first projecting $\mathrm{x}$ onto both dictionaries and evaluating the projection errors

$$
e^{ \pm}(\mathbf{x})=\arg \min _{\mathbf{z}^{ \pm}}\left\|\mathbf{D}^{ \pm} \mathbf{z}^{ \pm}-\mathbf{W} \mathbf{x}\right\|_{2}=\left\|\mathbf{P}^{ \pm} \mathbf{x}\right\|_{2},
$$

where $\mathbf{P}^{ \pm}=\mathbf{D}^{ \pm}\left(\mathbf{D}^{ \pm \mathrm{T}} \mathbf{D}^{ \pm}\right)^{-1} \mathbf{D}^{ \pm \mathrm{T}} \mathbf{W}$ are the $n \times n$ projection matrices. The point is sent to the left subtree if $e^{-}(\mathbf{x})<e^{+}(\mathbf{x})$, and to the right subtree otherwise. In practice, we only store the projection matrices $\mathbf{P}^{ \pm}$at each split node. Note that similar splitting functions report success in a classification context with much deeper trees in [19].

Optimization. To optimize the low-rank loss function (1) using gradient descent, the subgradient of the nuclear norm of a matrix can be computed as follows: Let $\mathbf{A}=$ $\mathbf{U} \boldsymbol{\Sigma} \mathbf{V}^{\mathrm{T}}$ be the SVD decomposition of the matrix A. Let $\hat{\mathbf{U}}$ and $\hat{\mathbf{V}}$ be the columns of $\mathbf{U}$ and $\mathbf{V}$ corresponding to eigenvalues larger than a predefined threshold. Following [17, 20], the subgradient of the nuclear norm can be evaluated in a simplified form as

$$
\partial\|\mathbf{A}\|_{*}=\hat{\mathbf{U}} \hat{\mathbf{V}}^{\mathrm{T}}
$$

Note that (1) is a D.C. (difference of convex functions) program; and the minimization is guaranteed to converge to a local minimum (or a stationary point), with the D.C. procedure detailed in [21,22].

Kernelization. A sufficient number of tree splits could potentially handle nonlinearity in data for classification. In this work, only very limited number of splits is preferred in each tree, e.g., depth 1 to 3 , to encourage short codes, which is insufficient in modeling data non-linearity well. Moreover, if we rely on tree splits in modeling nonlinearity, we may still obtain less confident class posteriors as explained. The low-rank loss in (1) is particularly effective when data approximately lie in linear subspaces [17]. To improve the ability of handling more generic data, an effective way is to map data points into an inner product space prior to optimize for low-rank loss.

Given a data point $\mathbf{y}$, we create a nonlinear map $\mathcal{K}(\mathbf{x})=\left(\kappa\left(\mathbf{x}, \mathbf{x}_{1}\right) ; \ldots ; \kappa\left(\mathbf{x}, \mathbf{x}_{n}\right)\right)$ by computing the inner product between $\mathbf{x}$ and a fixed set of $n$ points $\left\{\mathbf{x}_{1}, \ldots, \mathbf{x}_{n}\right\}$ 


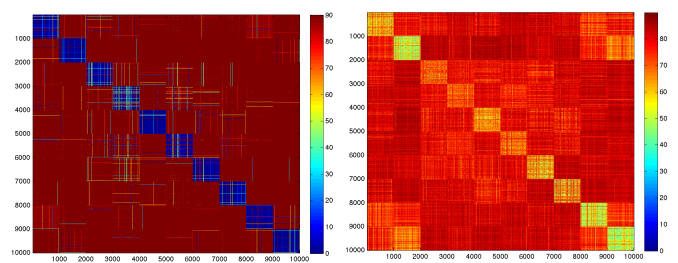

Fig. 3. Angles between the deep features learned for the validation set of CIFAR-10 using VGG16. (Left) with additional low-rank loss. (Right) with the standard softmax loss. With low-rank loss, the intra-class variations among features are collapsed and inter-class features are orthogonal, which are particularly preferred at each tree split node.

randomly drawn from the training set. The inner products are computed via the kernel function, $\kappa\left(\mathbf{x}, \mathbf{x}_{i}\right)=\varphi(\mathbf{x})^{\prime} \varphi\left(\mathbf{x}_{i}\right)$, which has to satisfy the Mercer conditions; note that no explicit representation for $\varphi$ is required. Examples of kernel functions include polynomial kernels $\kappa\left(\mathbf{y}, \mathbf{x}_{i}\right)=\left(\mathbf{x}^{\prime} \mathbf{x}_{i}+p\right)^{q}$ (with $p$ and $q$ being constants), and radial basis function (RBF) kernels $\kappa\left(\mathbf{x}, \mathbf{x}_{i}\right)=\exp \left(-\frac{\left\|\mathbf{x}-\mathbf{x}_{i}\right\|_{2}^{2}}{2 \sigma^{2}}\right)$ with variance $\sigma^{2}$. Given the data points $\mathbf{X}$, the set of mapped data is denoted as $\mathcal{K}(\mathbf{X}) \subseteq \mathbb{R}^{n}$. We now learn a weight matrix $\mathbf{W}$ minimizing,

$$
\min _{\mathbf{W}}\left\|\mathbf{W} \mathcal{K}\left(\mathbf{X}^{+}\right)\right\|_{*}+\left\|\mathbf{W} \mathcal{K}\left(\mathbf{X}^{-}\right)\right\|_{*}-\left\|\mathbf{W}\left[\mathcal{K}\left(\mathbf{X}^{+}\right), \mathcal{K}\left(\mathbf{X}^{-}\right)\right]\right\|_{*},
$$

Deep networks. While kernelization shows a simple yet effective non-linear mapping, we present a CNN-based weak learner now as the ultimate way in handling intricate data. With the gradient descent optimization discussed above, it is possible to implement the following function

$$
L=\left\|\mathbf{\Phi}\left(\mathbf{X}^{+}\right)\right\|_{*}+\left\|\mathbf{\Phi}\left(\mathbf{X}^{-}\right)\right\|_{*}-\left\|\left[\mathbf{\Phi}\left(\mathbf{X}^{+}\right), \mathbf{\Phi}\left(\mathbf{X}^{-}\right)\right]\right\|_{*},
$$

as a low-rank loss layer for general deep networks, where $\boldsymbol{\Phi}$ denotes the mapping from a deep network. From our experimental experience, the low-rank loss reports comparable performance as the standard softmax loss, while being used standalone as a classification loss for small classification problems. However, together with softmax, we observed consistent classification performance improvements over most popular CNN architectures and challenging datasets. As in Fig. 3, with low-rank loss, the intra-class variations among features are collapsed and inter-class features are orthogonal [23]. Such property is particularly beneficial at each tree split node.

\subsection{Information-theoretic code aggregation}

After training each random tree with the low-rank loss learner to produce consistent hashes for similar data points, we propose an information-theoretic approach to aggregate hashes across trees into a unique code for each data class. As labels are usually 
unavailable or only available for a small subset of data, unsupervised aggregation allows exploiting all available data. We also explain how labels, if available, can be further incorporated for supervised hash aggregation. Note that the code aggregation step is only learned once during training, no cost at testing.

Unsupervised aggregation. Consider a random forest consisting of $M$ trees of depth $d$; the hash codes obtained for $N$ training samples are denoted as $\mathcal{B}=\left\{\mathbf{B}_{i}\right\}_{i=1}^{M}$, with the $\mathbf{B}_{i} \in\{0,1\}^{\left(2^{d-1}\right) \times N}$ being the codes generated from the $i$-th tree, henceforth denoted as code blocks. Given the target hash code length $L$, our objective is to select $k$ code blocks $\mathbf{B}^{*}, k \leq L /\left(2^{d-1}\right)$, maximizing the mutual information between the selected and the remaining codes,

$$
\mathbf{B}^{*}=\arg \max _{\mathbf{B}:|\mathbf{B}|=k} I(\mathbf{B} ; \mathcal{B} \backslash \mathbf{B}) .
$$

A set function is said to be submodular if it has a diminishing return property, i.e., adding an element to a smaller set helps more than adding it to a larger set.

Lemma 2. $I(\mathbf{B} ; \mathcal{B} \backslash \mathbf{B})$ is submodular.

The general problem of maximizing submodular functions is NP-hard, by reduction from the max-cover problem. However, motivated by the sensor placement strategy in [24], we propose a very simple greedy algorithm to approximate the solution of (6). We start with $B=\emptyset$, and iteratively choose the next best code block $\mathbf{b}^{*}$ from $\mathcal{B} \backslash \mathbf{B}$ which provides a maximum increase in mutual information, i.e.,

$$
\begin{gathered}
\arg \max _{b^{*} \in \mathcal{B} \backslash \mathbf{B}} I\left(\mathbf{B} \cup \mathbf{b}^{*} ; \mathcal{B} \backslash\left(\mathbf{B} \cup \mathbf{b}^{*}\right)\right)-I(\mathbf{B} ; \mathcal{B} \backslash \mathbf{B}) \\
\quad=\arg \max _{b^{*} \in \mathcal{B} \backslash \mathbf{B}} H\left(\mathbf{b}^{*} \mid \mathbf{B}\right)-H\left(\mathbf{b}^{*} \mid \mathcal{B} \backslash\left(\mathbf{B} \cup \mathbf{b}^{*}\right)\right),
\end{gathered}
$$

where $H\left(\mathbf{b}^{*} \mid \mathbf{B}\right)$ denotes the conditional entropy. Intuitively, the first term $H\left(\mathbf{b}^{*} \mid \mathbf{B}\right)$ forces $\mathbf{b}^{*}$ to be most different from the already selected codes $\mathbf{B}$, and the second term $-H\left(\mathbf{b}^{*} \mid \mathcal{B} \backslash\left(\mathbf{B} \cup \mathbf{b}^{*}\right)\right)$ forces $\mathbf{b}^{*}$ to be most representative among the remaining codes. By defining a covariance matrix with the $i j$-th entry equal to $\exp \left(-\frac{d_{\mathbb{H}}\left(\mathbf{B}_{i}, \mathbf{B}_{j}\right)}{N}\right)$, with $d_{\mathbb{H}}$ being the Hamming distance, 77 can be efficiently evaluated in a closed form as detailed in [24]. It has been proved in [24,25] that the above greedy algorithm gives a polynomial-time approximation that is within $(1-1 / e)$ of the optimum, where $e$ is the the Napier's constant. Based on similar arguments as those in [24], the near-optimality of our approach can be guaranteed if the forest size $|\mathcal{B}|$ is sufficiently larger than $2 k$.

Supervised aggregation. When the class labels $C$ are available for the $N$ training samples, an upper bound on the Bayes error over hashing codes $\mathbf{B}$ is given by $\frac{1}{2}(H(C)-I(\mathbf{B} ; C))[26]$. This bound is minimized when $I(\mathbf{B} ; C)$ is maximized. Thus, discriminative hash codes can be obtained by maximizing

$$
\arg \max _{\mathbf{B}:|\mathbf{B}|=k} I(\mathbf{B} ; C) .
$$

Similarly to the unsupervised case, we maximize (8) using a greedy algorithm initialized with $\mathbf{B}=\emptyset$ and iteratively choosing the next best code block $\mathbf{b}^{*}$ from $\mathcal{B} \backslash \mathbf{B}$ which 
provides a maximum mutual information increase, i.e.,

$$
\arg \max _{\mathbf{b}^{*} \in \mathcal{B} \backslash \mathbf{B}} I\left(\mathbf{B} \cup \mathbf{b}^{*} ; C\right)-I(\mathbf{B} ; C),
$$

where $I(\mathbf{B} ; C)$ is evaluated as $I(\mathbf{B} ; C)=H(\mathbf{B})-\sum_{c=1}^{p} p(c) H(\mathbf{B} \mid c)$. Entropy measures here involve computation of probability density functions $p(\mathbf{B})$ and $p(\mathbf{B} \mid c)$, which can both be efficiently computed by counting the frequency of unique codes in $\mathbf{B}$. Note that the number of unique codes is usually very small due to the learned transformation step.

Semi-supervised aggregation. The above two aggregation models can be simply unified as

$$
\begin{array}{r}
\arg \max _{b^{*} \in \mathcal{B} \backslash \mathbf{B}}\left[I\left(\mathbf{B} \cup \mathbf{b}^{*} ; \mathcal{B} \backslash\left(\mathbf{B} \cup \mathbf{b}^{*}\right)\right)-I(\mathbf{B} ; \mathcal{B} \backslash \mathbf{B})\right] \\
+\lambda\left[I\left(\mathbf{B} \cup \mathbf{b}^{*} ; C\right)-I(\mathbf{B} ; C)\right] .
\end{array}
$$

The two terms here can be evaluated using different samples to exploit all labeled and unlabeled data. The parameter $\lambda$ in (10) is suggested to be estimated as the ratio between the maximal information gained from a code block to each respective criteria, i.e., $\lambda=\frac{\max _{i} I\left(\mathbf{B}_{i} ; \mathcal{B} \backslash \mathbf{B}_{i}\right)}{\max _{i} I\left(\mathbf{B}_{i} ; C\right)}$. Exploiting the diminishing return property, only the first greedily selected code block based on (7) and (9) need to be evaluated, which leads to an efficient process for finding $\lambda$. Selecting using only semantic information gives a hash model that is less robust, e.g., overfits to training data, than a model also concerning the actual code representation. As shown in the experiments, both unsupervised and supervised aggregation approaches promote unique codes for each class, with further improvements when both are unified.

\subsection{Multimodal hashing}

We can further extend ForestHash as a multimodal similarity learning approach. It is often challenging to enable similarity assessment across modalities, for example, searching a corpus consisting of audio, video, and text using queries from one of the modalities. The ForestHash framework can be easily extended for hashing data from multiple modalities into a single space.

At training, when multimodal data arrives at a tree split node, we simply enforce the same random class partition for all modalities, and learn for each modality a dictionary pair independently using the shared class partition. During training, only the splitting function of one dominant (usually most discriminant) modality is evaluated for each arriving data point; during testing, based on the modality of an arriving point, the corresponding splitting function acts independently. As shown in Section 3. ForestHash significantly outperforms state-of-the-art hashing approaches on cross-modality multimedia retrieval tasks.

\section{Experimental evaluation}

We present an experimental evaluation of ForestHash on image retrieval tasks using standard hashing benchmarks: the CIFAR-10 image dataset [27], the MNIST image 
dataset [28], and the Wikipedia image and document dataset [29]. CIFAR-10 is a challenging dataset of $60,00032 \times 32$ labeled color images with 10 different object categories, and each class contains 6,000 samples. MNIST consists of 8-bit grayscale handwritten digit images of " 0 " to " 9 " with 7,000 examples per class, and a total of 70,000 images.

\begin{tabular}{ccc}
\multicolumn{4}{c}{ CNN2 } \\
\hline 1 & Conv+ReLU+MaxPool & $5 \times 5 \times 3 \times 64$ \\
2 & Conv+ReLU+MaxPool & $5 \times 5 \times 64 \times 32$ \\
3 & FC & output: 256 \\
\hline & CNN4 & \\
\hline & Conv+ReLU+MaxPool & $5 \times 5 \times 3 \times 64$ \\
2 & Conv+ReLU+MaxPool & $5 \times 5 \times 64 \times 64$ \\
3 & Conv+ReLU+MaxPool & $5 \times 5 \times 64 \times 64$ \\
4 & Conv+ReLU+MaxPool & $5 \times 5 \times 64 \times 64$ \\
5 & FC & output: 256 \\
\hline
\end{tabular}

Table 1. Network structures of light-weight CNN learners.

\begin{tabular}{l|cccc}
\hline Method & 12-bit & 24-bit & 36-bit & 48-bit \\
\hline \hline LSH [1] & 0.13 & 0.14 & 0.14 & 0.15 \\
SH [3] & 0.13 & 0.13 & 0.14 & 0.13 \\
ITQ 30] & 0.11 & 0.11 & 0.11 & 0.12 \\
CCA-11Q 30 & 0.17 & 0.20 & 0.21 & 0.22 \\
MLH [31] & 0.18 & 0.20 & 0.21 & 0.21 \\
BRE 32] & 0.16 & 0.16 & 0.17 & 0.17 \\
KSH J & 0.29 & 0.37 & 0.40 & 0.42 \\
CNNH 33] & 0.54 & 0.56 & 0.56 & 0.56 \\
DLBHC 34] & 0.55 & 0.58 & 0.58 & 0.59 \\
DNNH 35 & 0.57 & 0.59 & 0.59 & 0.59 \\
DSH 8 & 0.62 & 0.65 & 0.66 & 0.68 \\
\hline ForestHash-CNN2 & 0.61 & 0.75 & 0.78 & 0.80 \\
ForestHash-CNN4 & 0.70 & 0.80 & 0.82 & 0.84 \\
ForestHash-VGG16 & $\mathbf{0 . 7 6}$ & $\mathbf{0 . 8 2}$ & $\mathbf{0 . 8 6}$ & $\mathbf{0 . 8 9}$ \\
\hline
\end{tabular}

Table 2. Retrieval performance (mAP) of different hashing methods on CIFAR-10. All methods use the $32 \times 32$ RGB images as input.

As discussed in Section 2.3. a low-rank weak learner at each tree split node is allowed in various implementations. Without particular specification, a 256-dimensional RBF kernelization is assumed. We use the CNN suffix when using a light-weight CNN as weak learner. Table 1 shows two network structures of light-weight CNN learners, CNN2 and CNN4, adopted in experiments. Unless otherwise specified, 128 trees are trained and semi-supervised aggregation are used (with only training data).

Note that a shallow tree is preferred; and a deeper tree $(d \geq 8)$ becomes less preferred for (fast) retrieval, and loses the robustness gained from randomness. A tree of depth 2 is assumed by default in this section. In practice, the choice of tree depth also depends of the target code length and the level of parallelism supported, as each hash tree can be trained and deployed independently in parallel. 


\begin{tabular}{l|ccc}
\hline Method & depth & params & CIFAR-10 \\
\hline \hline Network in Network [36] & - & - & 10.41 \\
All-CNN [37] & - & - & 9.08 \\
Deeply Supervised Net [38] & - & - & 9.69 \\
FractalNet [39] & 21 & $38.6 \mathrm{M}$ & 10.18 \\
ResNet ( 40) & 110 & $1.7 \mathrm{M}$ & 13.63 \\
ResNet with Stochastic Depth 40] & 110 & $1.7 \mathrm{M}$ & 11.66 \\
\hline ResNet (pre-activation) [41] & 164 & $1.7 \mathrm{M}$ & 11.26 \\
& 1001 & $10.2 \mathrm{M}$ & 10.56 \\
\hline ForestHash CNN2 128-bit & $2(\times 64)$ & $0.58 \mathrm{M}(\times 64)$ & 20.3 \\
ForestHash CNN4 128-bit & $4(\times 64)$ & $0.38 \mathrm{M}(\times 64)$ & 16.47 \\
ForestHash VGG16 128-bit & $16(\times 64)$ & $20.1 \mathrm{M}(\times 64)$ & 11.03 \\
\hline
\end{tabular}

Table 3. Error rates (\%) on CIFAR-10 image classification benchmark. ForestHash performs at the level of other state-of-the-art image classification techniques while utilizing a very compact 128-bit only representation.

\begin{tabular}{l|ll||ll}
\hline & \multicolumn{2}{|c||}{ radius = 0 } & \multicolumn{2}{c}{ radius $\leq 2$} \\
\hline Method & \multicolumn{2}{|c||}{ Precision Recall } & \multicolumn{2}{|c}{ Precision Recall } \\
\hline \hline SH [3 & 5.90 & 0.01 & 21.00 & 0.25 \\
KSH 5 & 8.50 & 0.07 & 21.41 & 0.66 \\
AGH1 6 & 29.48 & 0.21 & 30.55 & 0.41 \\
AGH2 & 29.92 & 0.24 & 30.13 & 0.58 \\
SparseHash [4 & 16.65 & 0.05 & 32.69 & 1.81 \\
\hline ForestHash (rand) & 31.37 & 2.74 & 32.25 & 4.90 \\
ForestHash (unsup) & 34.02 & 3.65 & 34.55 & 6.40 \\
ForestHash (sup) & 33.86 & 3.33 & 34.02 & 5.21 \\
ForestHash (semi) & $\mathbf{3 4 . 0 5}$ & $\mathbf{4 . 1 2}$ & 33.73 & $\mathbf{7 . 2 9}$ \\
\hline \hline ForestHash CNN4-softmax & 22.72 & 0.33 & 34.27 & 1.52 \\
ForestHash CNN2-softmax & 23.00 & 0.42 & 32.13 & 1.56 \\
ForestHash CNN4 & 28.66 & 0.86 & $\mathbf{3 8 . 6 0}$ & 2.88 \\
ForestHash CNN2 & 29.30 & 1.78 & 38.29 & 4.68 \\
\hline
\end{tabular}

Table 4. Retrieval performance (\%) of different hashing methods (48-bit codes) on CIFAR-10 using reduced training. The methods on the top two groups use GIST features. For reference, the bottom group shows the performance of ForestHash with CNN features extracted from the 32x32 RGB images.

\subsection{Image retrieval}

We first adopt a CIFAR-10 protocol popular among many deep-learning based hashing methods, e.g., DSH [8], where the official CIFAR-10 train/test split is used; namely, 50,000 images are used as the training and the gallery, and 10,000 images as the query. Table 2 reports the retrieval performance comparisons with multiple hashing methods 4 ForestHash with a simplest two-layer learner CNN2 in Table 1 already significantly outperforms state-of-the-art methods. Given such large size of training set, retrieval performance increases using more complex network structures as learners, e.g., CNN4 or VGG16 over CNN2.

The superior retrieval performance of the ForestHash codes in Table 2 can be easily explained by both the low-rank loss properties in Figure 3 and the boosting effect of the random forest in Figure 4. ForestHash shows a principled and robust procedure to train and deploy in parallel an ensemble of light-weight CNNs, instead of simply going deeper. As shown in Table 3. ForestHash performs at the level of other state-

\footnotetext{
${ }^{4}$ Results are taken from the respective papers.
} 


\begin{tabular}{|c|c|c|c|c|c|c|c|c|}
\hline & \multirow[b]{2}{*}{$\begin{array}{l}\text { Test } \\
\text { time }(\mu \mathrm{s})\end{array}$} & \multicolumn{2}{|c|}{6,000 samples per class } & \multicolumn{3}{|c|}{100 samples per class } & \multicolumn{2}{|c|}{30 samples per class } \\
\hline & & $\begin{array}{l}\text { Train } \\
\text { time (s) }\end{array}$ & Prec. Rec. & $\begin{array}{l}\text { Train } \\
\text { time }(\mathrm{s})\end{array}$ & Prec. & Rec. & $\begin{array}{l}\text { Train } \\
\text { time }(\mathrm{s})\end{array}$ & Prec. Rec. \\
\hline$\overline{\mathrm{HDML}} \overline{10}$ & \begin{tabular}{|l|l}
10 &
\end{tabular} & 93780 & 92.9460 .44 & 1505 & 62.52 & 2.19 & 458 & 24.280 .21 \\
\hline FastHash 42 & 115 & 865 & 84.7076 .60 & 213 & 73.32 & 33.04 & 151 & $57.08 \quad 11.77$ \\
\hline TSH 43 & 411 & 164325 & 86.303 .17 & 21.08 & 74.00 & 5.19 & 2.83 & 56.863 .94 \\
\hline ForestHash & 57 & 24.20 & 86.5346 .30 & 4.19 & 84.98 & 45.00 & 1.43 & 79.3842 .27 \\
\hline ForestHash CNN2 & 13 & 81.6 & 97.9995 .99 & 7 & 94.24 & 74.02 & 2.69 & 89.5646 .36 \\
\hline
\end{tabular}

Table 5. 36-bit retrieval performance (\%) on MNIST (rejection hamming radius 0) using different training set sizes. Test time is the average binary encoding time in microseconds $(\mu \mathrm{s})$.

\begin{tabular}{|c|c|c|c|c|c|c|c|c|c|}
\hline & \begin{tabular}{|l|} 
ForestHash \\
CNN2
\end{tabular} & ForestHash & USPLH & 44 & $\mathrm{SH} 3$ & KLSH 2 & SIKH 45 & AGH1 6 & AGH2 \\
\hline 24 bits & 99.63 & 82.99 & 46.99 & & 26.99 & 25.55 & 19.47 & 499.97 & 67.38 \\
\hline 48 bits & 99.68 & 86.09 & 49.30 & & 24.53 & 30.49 & 19.72 & 39.71 & 64.10 \\
\hline
\end{tabular}

Table 6. Mean average precision (mAP in \%) in percent of Hamming ranking on MNIST.

of-the-art image classification techniques, e.g., ResNet, while utilizing a 128-bit only representation.

We further experiment with CIFAR-10 using reduced size of training with both handcrafted feature and deep features. We adopt the same setup as in [4,5] for the image retrieval experiments: we only used 200 images from each class for training; and for testing, a disjoint test set of 1,000 images are evenly sampled from ten classes, to query the remaining 59,000 images. Images are used as inputs for ForestHash with CNN learners, and 384-dimensional GIST descriptors are used for other compared methods, including ForestHash with an RBF kernel.

Table 4 summarizes the retrieval performance of various methods on CIFAR-10 at reduced training using the mean precision and recall for Hamming radius 0 and 2 hash look-up. For the compared methods SH, KSH, AGH1 and AGH2, we use the code provided by the authors; while for SparseHash, we reproduce the results from [4]. $\mathrm{SH}$ is unsupervised, while the rest of the hashing schemes are all supervised. We report the performance of ForestHash using the random, unsupervised, supervised, and semi-supervised hash aggregation schemes, respectively. We observe that the proposed information-theoretic code aggregation provides an effective way to combine hashes from different trees, and showing further benefits to unify both unsupervised and supervised aggregation. We also observe that using softmax loss only for CNN learners leads to performance degradation. At reduced training, more complex learner structures show no obvious advantage. In general, the proposed ForestHash shows significantly higher precision and recall compared to other methods.

The supervised hashing methods HDML [10], TSH [43], and FastHash [42] report excellent performance, where HDML is a deep learning based hashing method, and FastHash is a boosted trees based method. We adopt the experimental setting from [10], i.e., a $60 \mathrm{~K}$ training set and a disjoint $10 \mathrm{~K}$ query set split on the MNIST data. Each hashing method is assessed by the retrieval precision and recall at radius 0 . As shown in Table 5, using all 60K training samples, ForestHash with an RBF kernel shows comparable performance as HDML and FastHash, and better than TSH. ForestHash with a two-layer CNN significantly outperform all compared methods. We further assume 


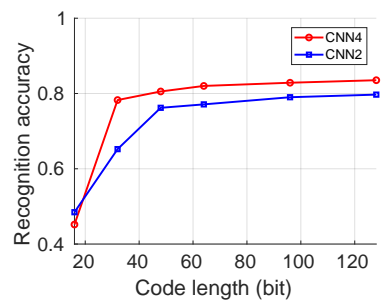

Fig. 4. The forest boosting effect using ForestHash codes. ForestHash shows a principled and robust procedure to train and deploy in parallel an ensemble of light-weight CNNs, instead of simply going deeper.

\begin{tabular}{|c|c|c|c|c|c|c|c|}
\hline $\begin{array}{l}\text { ForestHash } \\
\text { (64-bit) }\end{array}$ & $\begin{array}{l}\text { ForestHash } \\
\text { (36-bit) }\end{array}$ & CM-SSH $\overline{46}$ & CM $\overline{29}$ & SM 29 & SCM 29 & MM-NN $\overline{9}$ & CM-NN $\overline{9}$ \\
\hline 50.8 & 45.5 & 18.4 & 19.6 & 22.3 & 22.6 & 27.4 & 25.8 \\
\hline
\end{tabular}

Table 7. Cross-modality image retrieval using text queries on the Wiki dataset ( $\mathrm{mAP}$ in \%).

labels are only available for a small subset of data, which is often the case for a retrieval system. When the number of labeled samples reduces to 100 and 30 per class respectively (instead of 6,000), the retrieval performance of other deep learning and boosted tree-based hashing degrades dramatically, as those methods require a dense training set to learn a rich set of parameters. Due to the subspace assumption behind the low-rank loss, which are known to be robust in the regime with few labeled training examples per class [47], ForestHash significantly outperforms state-of-the-art methods for such reduced training cases. Note that the training and hashing time of ForestHash reported here is the time for one tree, in order to emphasize the fact that different trees are trained and deployed independently and can easily be done in parallel

More experiments were conducted on MNIST following [6], enabling the comparison with more hashing methods for which we have no implementation accessible. We split the MNIST dataset into a training set containing 69,000 samples and a disjoint query set of 1,000 samples. Table 6 reports the Hamming ranking performance measured by the Mean Average Precision (mAP) (performance of other methods is reproduced from [6]). For both code lengths, the proposed ForestHash significantly outperforms other hashing methods.

\subsection{Cross-modality retrieval}

We performed a cross-modality retrieval experiment following [9] 29] on the Wikipedia dataset. The purpose is to demonstrate that ForestHash natively supports cross-modality, though not being designed for. The Wikipedia dataset contains a total of 2866 documents. These are article-image pairs, annotated with a label from 10 semantic classes. To enable a fair comparison, we adopted the provided features for both images and text from [29]. Table 7 shows the mean average precision scores for the cross-modality image retrieval using text queries. The proposed ForestHash significantly outperforms state-of-the-art hashing approaches on cross-modality multimedia retrieval tasks. Note 


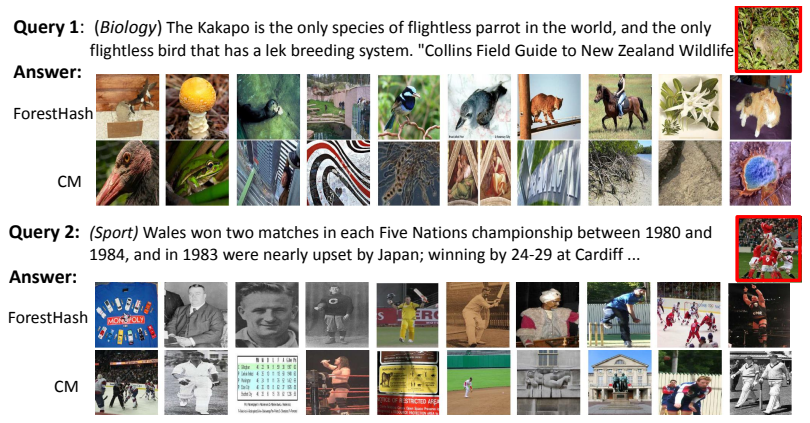

Fig. 5. Two examples of text queries and the top-10 images retrieved by ForestHash and CM [29]. Note that only text information are used to compose each query, and images are retrieved from the same category of the query text.

that MM-NN and CM-NN [9] in Table 7 are both deep learning motivated hashing methods. Two examples of cross-modality text queries and the top-10 images retrieved are shown in Figure 5, using ForestHash and CM [29]. Note that only text information is used to compose a query, and ForestHash retrieves images from the same category of the query text. ForestHash significantly outperforms CM with codes at least $10 \times$ shorter.

\section{Conclusion}

Considering the importance of compact and computationally efficient codes, we introduced a random forest semantic hashing scheme, extending random forest beyond classification and for large-scale multimodal retrieval of incommensurable data. The proposed scheme consists of a forest with random class grouping, low-rank loss, and an information-theoretic code aggregation scheme. Using the matrix nuclear norm as the optimization criterion, the low-rank loss simultaneously reduces variations within the classes and increases separations between the classes. Thus, hash consistency (similarity) among similar samples is enforced in a random tree. The information-theoretic code aggregation scheme provides a nearly optimal way to combine hashes generated from different trees, producing a unique code for each sample category, and is applicable in training regimes ranging from totally unsupervised to fully supervised. Note that the proposed framework combines in a fundamental fashion kernel methods, random forests, CNNs, and hashing. Our method shows exceptional effectiveness in preserving similarity in hashes, and significantly outperforms state-of-the-art hashing methods in large-scale single- and multi-modal retrieval tasks.

\section{Acknowledgements}

Work partially supported by AFOSR, ARO, NGA, NSF, ONR. José Lezama was supported by ANII (Uruguay) grant PD_NAC_2015_1_108550. 


\section{References}

1. Gionis, A., Indyk, P., Motwani, R.: Similarity search in high dimensions via hashing. In: Proc. of International Conference on Very Large Data Bases. (1999)

2. Kulis, B., Grauman, K.: Kernelized locality-sensitive hashing for scalable image search. In: Proc. International Conference on Computer vision. (2009)

3. Weiss, Y., Torralba, A., Fergus, R.: Spectral hashing. In: Advances in Neural Information Processing Systems. (2009)

4. Masci, J., Bronstein, A.M., Bronstein, M.M., Sprechmann, P., Sapiro, G.: Sparse similaritypreserving hashing. In: International Conference on Learning Representations, Banff, Canada (April, 2014)

5. Liu, W., Wang, J., Ji, R., Jiang, Y., Chang, S.: Supervised hashing with kernels. In: Proc. IEEE Computer Society Conf. on Computer Vision and Patt. Recn. (June 2012)

6. Liu, W., Wang, J., Chang, S.: Hashing with graphs. In: International Conference on Machine Learning. (2011)

7. Zhang, D., Wang, J., Cai, D., Lu, J.: Self-taught hashing for fast similarity search. In: Proc. of International Conference on Research and Development in Information Retrieval. (2010)

8. Liu, H., Wang, R., Shan, S., Chen, X.: Deep supervised hashing for fast image retrieval. In: Proc. IEEE Computer Society Conf. on Computer Vision and Patt. Recn. (2016)

9. Masci, J., Bronstein, M.M., Bronstein, A.M., Schmidhuber, J.: Multimodal similaritypreserving hashing. IEEE Trans. on Patt. Anal. and Mach. Intell. 36(4) (2014) 824-830

10. Norouzi, M., Fleet, D.J., Salakhutdinov, R.: Hamming distance metric learning. In: Advances in Neural Information Processing Systems. (2012)

11. Breiman, L.: Random forests. Machine Learning 45(1) (2001) 5-32

12. Criminisi, A., Shotton, J.: Decision Forests for Computer Vision and Medical Image Analysis. Springer (2013)

13. Shotton, J., Girshick, R., Fitzgibbon, A., Sharp, T., Cook, M., Finocchio, M., Moore, R., Kohli, P., Criminisi, A., Kipman, A., Blake, A.: Efficient human pose estimation from single depth images. IEEE Trans. on Patt. Anal. and Mach. Intell. 35(12) (2013)

14. Gall, J., Lempitsky, V.: Class-specific hough forests for object detection. In: Proc. IEEE Computer Society Conf. on Computer Vision and Patt. Recn. (2009)

15. Quinlan, J.R.: C4.5: Programs for Machine Learning. Morgan Kaufmann Publishers Inc. (1993)

16. Fazel, M.: Matrix Rank Minimization with Applications. PhD Thesis, Stanford University (2002)

17. Qiu, Q., Sapiro, G.: Learning transformations for clustering and classification. Journal of Machine Learning Research 16 (2015) 187-225

18. Aharon, M., Elad, M., Bruckstein, A.: K-SVD: An algorithm for designing overcomplete dictionaries for sparse representation. IEEE Trans. on Signal Processing 54(11) (Nov. 2006) 4311-4322

19. Qiu, Q., Sapiro, G.: Learning transformations for classification forests. In: ICLR. (2014)

20. Watson, G.A.: Characterization of the subdifferential of some matrix norms. Linear Algebra and Applications 170 (1992) 1039-1053

21. Sriperumbudur, B.K., Lanckriet, G.R.G.: A proof of convergence of the concave-convex procedure using zangwill's theory. Neural Computation 24(6) (2012) 1391-1407

22. Yuille, A.L., Rangarajan, A.: The concave-convex procedure. Neural Computation 4 (2003) 915-936

23. Lezama, J., Qiu, Q., Musé, P., Sapiro, G.: Olé: Orthogonal low-rank embedding, a plug and play geometric loss for deep learning. In: Proc. IEEE Computer Society Conf. on Computer Vision and Patt. Recn. (2018) 
24. Krause, A., Singh, A., Guestrin, C.: Near-optimal sensor placements in Gaussian processes: Theory, efficient algorithms and empirical studies. Journal of Machine Learning Research (9) (2008) 235-284

25. Nemhauser, G., Wolsey, L., Fisher, M.: An analysis of approximations for maximizing submodular set functions. Mathematical Programming 14(1) (1978) 265-294

26. Hellman, M.E., Raviv, J.: Probability of error, equivocation, and the Chernoff bound. IEEE Trans. on Info. Theory 16 (1979) 368-372

27. Krizhevsky, A.: Learning multiple layers of features from tiny images. Technical report (2009)

28. Lecun, Y., Bottou, L., Bengio, Y., Haffner, P.: Gradient-based learning applied to document recognition. Proceedings of the IEEE 86(11) (Nov 1998) 2278-2324

29. Rasiwasia, N., Costa Pereira, J., Coviello, E., Doyle, G., Lanckriet, G.R., Levy, R., Vasconcelos, N.: A new approach to cross-modal multimedia retrieval. In: Proceedings of the International Conference on Multimedia. (2010)

30. Gong, Y., Lazebnik, S.: Iterative quantization: A procrustean approach to learning binary codes. In: Proc. IEEE Computer Society Conf. on Computer Vision and Patt. Recn. (2011)

31. Norouzi, M., Fleet, D.J.: Minimal loss hashing for compact binary codes. In: International Conference on Machine Learning. (2011)

32. Kulis, B., Darrell, T.: Learning to hash with binary reconstructive embeddings. In: Advances in Neural Information Processing Systems. (2009)

33. Xia, R., Pan, Y., Lai, H., Liu, C., Yan, S.: Supervised hashing for image retrieval via image representation learning. In: Proceedings of the Twenty-Eighth AAAI Conference on Artificial Intelligence. (2014)

34. Lin, K., Yang, H.F., Hsiao, J.H., Chen, C.S.: Deep learning of binary hash codes for fast image retrieval. In: 2015 IEEE Conference on Computer Vision and Pattern Recognition Workshops (CVPRW). (2015)

35. Lai, H., Pan, Y., Liu, Y., Yan, S.: Simultaneous feature learning and hash coding with deep neural networks. In: Proc. IEEE Computer Society Conf. on Computer Vision and Patt. Recn. (2015)

36. Lin, M., Chen, Q., Yan, S.: Network In Network. ICLR (2014)

37. Springenberg, J.T., Dosovitskiy, A., Brox, T., Riedmiller, M.A.: Striving for simplicity: The all convolutional net. CoRR abs/1412.6806 (2014)

38. Lee, C., Xie, S., Gallagher, P.W., Zhang, Z., Tu, Z.: Deeply-supervised nets. In: AISTATS. (2015)

39. Larsson, G., Maire, M., Shakhnarovich, G.: Fractalnet: Ultra-deep neural networks without residuals. In: ICLR. (2017)

40. Huang, G., Sun, Y., Liu, Z., Sedra, D., Weinberger, K.Q.: Deep networks with stochastic depth. In: Proc. European Conference on Computer Vision. (2016)

41. He, K., Zhang, X., Ren, S., Sun, J.: Identity mappings in deep residual networks. In: Proc. European Conference on Computer Vision. (2016) 630-645

42. Lin, G., Shen, C., Shi, Q., van den Hengel, A., Suter, D.: Fast supervised hashing with decision trees for high-dimensional data. In: Proc. IEEE Computer Society Conf. on Computer Vision and Patt. Recn. (2014)

43. Lin, G., Shen, C., Suter, D., van den Hengel, A.: A general two-step approach to learningbased hashing. In: Proc. International Conference on Computer vision. (2013)

44. Wang, J., Kumar, S., Chang, S.F.: Sequential projection learning for hashing with compact codes. In: International Conference on Machine Learning, Haifa, Israel (2010)

45. Raginsky, M., Lazebnik, S.: Locality-sensitive binary codes from shift-invariant kernels. In: Advances in Neural Information Processing Systems. (2010) 
46. Bronstein, M., Bronstein, A., Michel, F., Paragios, N.: Data fusion through cross-modality metric learning using similarity-sensitive hashing. In: Proc. IEEE Computer Society Conf. on Computer Vision and Patt. Recn. (June 2010)

47. Bengio, Y., Courville, A., Vincent, P.: Representation learning: A review and new perspectives. IEEE Trans. on Patt. Anal. and Mach. Intell. 35(8) (2013) 1798-1828 